\title{
IPHAS J025827.88+635234.9 and IPHAS J051814.33+294113.0: Two probable eclipsing intermediate polars
}

\author{
Arti Joshi*, Jeewan Chandra Pandey
}

Aryabhatta Research Institute of Observational Sciences (ARIES), Nainital-263002, India

\begin{abstract}
We present photometry in the R-band and linear polarimetry of two cataclysmic variables, namely IPHAS J025827.88+635234.9 and IPHAS J051814.33+294113.0. The data were obtained from 1-m class telescopes of the Aryabhatta Research Institute of Observational Sciences (ARIES; Nainital, India). In addition to the deep eclipse, strong short-period oscillations are also found. We derived a pulse period of $(1203 \pm 25) \mathrm{s}$ for IPHAS J025827.88+635234.9 and (3277 \pm 81$)$ s for IPHAS J051814.33+294113.0. The presence of both orbital and spin modulations in these systems indicate that they belong to a class of intermediate polars. The full width at half depth of the eclipse is also found to be variable from epoch to epoch for IPHAS J025827.88+635234.9. The presence of a variable linear polarization of high value in these two sources indicates that these systems possess a strong magnetic field.
\end{abstract}

\section{Introduction}

Cataclysmic variables (CVs) are evolved, semi-detached interacting binaries containing a magnetic white dwarf (WD) as a primary star, which accretes material from a Roche lobe-filling secondary red dwarf star through the inner Lagrangian point (Warner 1995). Based on the magnetic field strength of the white dwarf, CVs are broadly classified into two categories: non-magnetic CVs and magnetic CVs. If the magnetic field strength of the WD is weak $(B<0.01 \mathrm{MG})$ such systems are referred to as non-magnetic $\mathrm{CVs}$, where the accretion occurs through an accretion disc. Magnetic CVs are further classified into two categories: polars and intermediate polars (IPs). Polars are synchronized binaries ( $P_{\Omega}=P_{\omega}$; where $\Omega$ and $\omega$ are the orbital and spin frequencies, respectively) with magnetic field strengths above $10 \mathrm{MG}$, whereas IPs are asynchronous systems $\left(P_{\omega}<P_{\Omega}\right)$ with white dwarf magnetic field strengths below $10 \mathrm{MG}$. The weaker magnetic fields and wide orbits of the IPs allow the material from the companion red dwarf star to form an accretion disk around the WD. But the inner part of the disk is disrupted into accretion curtains and columns before the material is dumped to the magnetic poles of the WD. The spin to orbital period ratios for IPs are in the range of $0.25 \geq$ $\mathrm{P}_{\omega} / \mathrm{P}_{\Omega} \geq 0.01$ and $\mathrm{P}_{\Omega} \geq 3 \mathrm{hr}$. There are two other systems, namely Paloma and V697 Sco, which are described as "nearly synchronous IPs", with $P_{\omega}=(0.7-0.9) P_{\Omega}$ (Norton et al. 2004; Schwarz et al. 2007; Joshi et al. 2016). Some IPs are also referred to as "EX Hya-like systems", where $\mathrm{P}_{\omega} / \mathrm{P}_{\Omega}$

\footnotetext{
*E-mail: arti@aries.res.in
} 
Table 1: Log of observations for photometric and polarimetric data of IPHAS J0258 and IPHAS J0518. For each object and each observation date, we give the telescope, instrument and filter used for the observations along with the exposure times and the total length of the data strings.

\begin{tabular}{lcccccc}
\hline Object & Date & Telescope & Instrument & Filter & Exp & Length \\
\hline IPHAS J0258 & $05 / 11 / 2015$ & $1.3-\mathrm{m}$ DFOT & $512 \times 512$ & $\mathrm{R}$ & $40 \mathrm{~s}$ & $3.6 \mathrm{hrs}$ \\
& $20 / 11 / 2015$ & $1.3-\mathrm{m}$ DFOT & $512 \times 512$ & $\mathrm{R}$ & $20 \mathrm{~s}$ & $2.9 \mathrm{hrs}$ \\
& $17 / 10 / 2015$ & $1.04-\mathrm{m} \mathrm{ST}$ & AIMPOL & $\mathrm{R}$ & $100 \mathrm{~s}$ & $3.2 \mathrm{hrs}$ \\
& $13 / 11 / 2015$ & $1.04-\mathrm{m} \mathrm{ST}$ & AIMPOL & $\mathrm{R}$ & $100 \mathrm{~s}$ & $3.6 \mathrm{hrs}$ \\
& $14 / 11 / 2015$ & $1.04-\mathrm{m} \mathrm{ST}$ & AIMPOL & $\mathrm{R}$ & $100 \mathrm{~s}$ & $4.4 \mathrm{hrs}$ \\
& $16 / 12 / 2015$ & $1.04-\mathrm{m} \mathrm{ST}$ & AIMPOL & $\mathrm{R}$ & $100 \mathrm{~s}$ & $3.2 \mathrm{hrs}$ \\
IPHAS J0518 & $20 / 11 / 2015$ & $1.3-\mathrm{m}$ DFOT & $512 \times 512$ & $\mathrm{R}$ & $100 \mathrm{~s}$ & $5.0 \mathrm{hrs}$ \\
& $20 / 10 / 2015$ & $1.04-\mathrm{m} \mathrm{ST}$ & AIMPOL & $\mathrm{R}$ & $100 \mathrm{~s}$ & $3.3 \mathrm{hrs}$ \\
& $13 / 11 / 2015$ & $1.04-\mathrm{m} \mathrm{ST}$ & AIMPOL & $\mathrm{R}$ & $100 \mathrm{~s}$ & $4.0 \mathrm{hrs}$ \\
& $14 / 11 / 2015$ & $1.04-\mathrm{m} \mathrm{ST}$ & AIMPOL & $\mathrm{R}$ & $100 \mathrm{~s}$ & $5.0 \mathrm{hrs}$ \\
\hline
\end{tabular}

$>0.1$ and $\mathrm{P}_{\Omega}<3 \mathrm{hr}$ (Allan et al. 1998; Hellier et al. 2002; Andronov \& Breus 2013). The orbital and spin period distribution of magnetic CVs shows that the majority of the IPs have orbital periods longer than the period gap of 2-3 hrs (Norton et al. 2004, 2008; Scaringi et al. 2010).

The cyclotron emission from near the surface of the WD is highly polarized in the presence of the strong magnetic field. As a large fraction of the optical flux in polars comes from this region, therefore showing large polarization (both linear and circular), while only a few IPs (BG CMi, PQ Gem, V2400 Oph, V405 Aur, and V2306 Cyg) have been found to emit polarized light. A linear polarization pulse was also detected in many of the polars (Piirola et al. 1990; Wickramasinghe et al. 1991) but not in the IPs, with the exception of RE $0751+14$.

In this work, we present analyses of optical photometric and polarimetric observations of the objects IPHAS J025827.88+635234.9 (hereafter 'IPHAS J0258') and IPHAS J051814.33+294113.0 (hereafter 'IPHAS J0518'). These two sources were identified as CVs by Witham et al. (2007) with IPHAS (Isaac Newton Telescope Photometric $\mathrm{H} \alpha$ Survey of the northern Galactic plane). This identification was based on the virtue of the broad Balmer and Helium emission lines in their spectra. The prominent He II emission line at $468.57 \mathrm{~nm}$ along with the $\mathrm{H} \beta$ emission line are also present in the spectra of IPHAS J0258 and IPHAS J0518, which is a common characteristic of magnetic CVs (Witham et al. 2007). Using optical photometry, Kozhevnikov (2014) has derived an orbital period of $5.88 \mathrm{hr}$ for IPHAS J0258. The distance of IPHAS J0258 was estimated to be in the range of 660730 pc (Ak et al. 2007; Kozhevnikov 2014). Witham et al. (2007) have determined four possible orbital periods for IPHAS J0518 $(0.2383 \mathrm{~d}, 0.2203 \mathrm{~d}, 0.2595 \mathrm{~d}$, and $0.2049 \mathrm{~d})$ using radial velocity measurements of the $\mathrm{H} \alpha$ emission line. In this paper, we present recent photometric and polarimetric observations of these two probable magnetic CVs. The paper is organized as follows. In the next section, we present the observations and data reduction. Section 3 contains analyses and the results of the optical photometric and polarimetric observations. Finally, we present a discussion and our conclusions in Sect. 4.

\section{Observations And Data Reduction}

IPHAS J0258 and IPHAS J0518 were observed by 1-m class telescopes of ARIES (Nainital, India). A $\log$ of the observations is given in Table 1. Details about the telescopes and their reduction procedures 

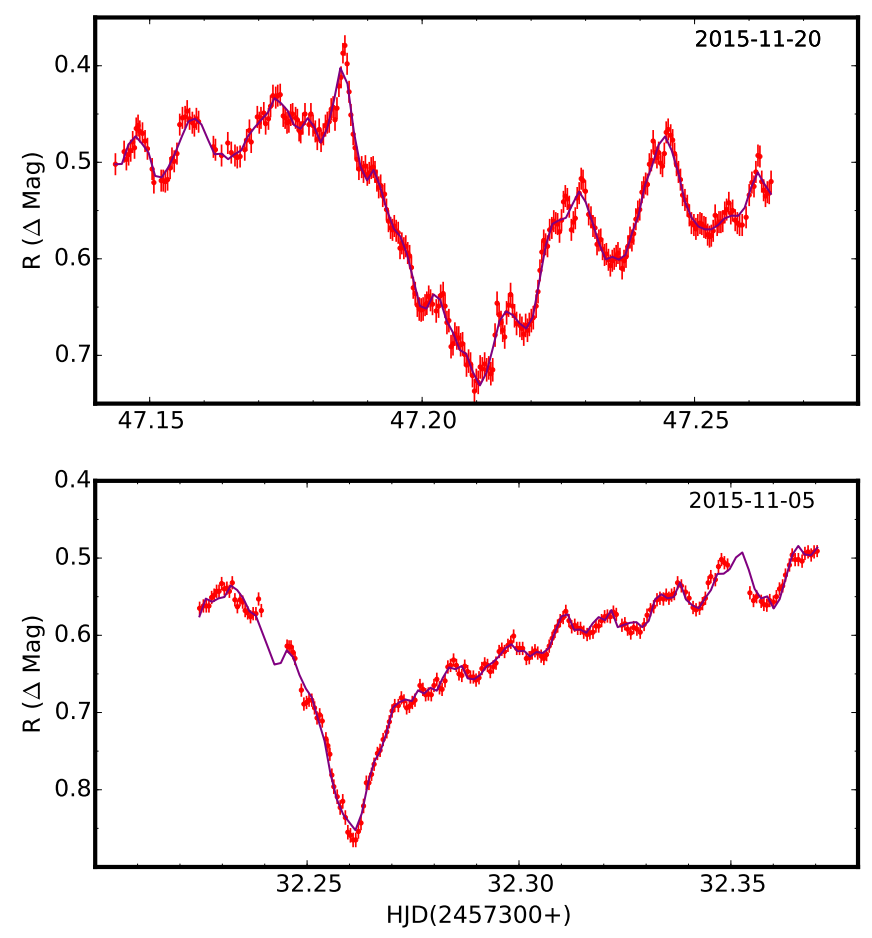

Figure 1: R-band light curve of IPHAS J0258 (red points with error bars) along with the 15 order trigonometric polynomial fit (puple line). The observation dates are mentioned at the top of each panel.

are given below.

\subsection{Photometry}

R-band photometric observations of IPHAS J0258 and IPHAS J0518 were obtained in November 2015 using the 1.3-m Devasthal Fast Optical Telescope located at Devasthal, India (Sagar et al. 2011). The 1.3-m telescope has Ritchey-Chretien (RC) optics with a f/4 beam at the Cassegrain focus. The telescope is equipped with a $512 \times 512$ Andor CCD (read noise $=6.1 e^{-}(\mathrm{rms})$ and gain $=1.4 e^{-} / \mathrm{ADU}$ at $1 \mathrm{MHz}$ readout speed) with a pixel size of 16 micron and a plate scale of 0.63 arcsec per pixel. The field of view (FOV) of the CCD is $\sim 5$ arcmin on the sky. Several bias and twilight sky flat frames were also taken during the observing runs. The pre-processing (i.e. bias subtraction and flat fielding) and R-band differential photometry were performed using IRAF ${ }^{1}$ software.

\subsection{Linear Polarization}

Linear polarimetric observations of IPHAS J0258 and IPHAS J0518 were obtained using the Aries Imaging Polarimeter (AIMPOL; Rautela, Joshi \& Pandey 2004), mounted on the 1.04-m Sampurnanand Telescope (ST) at ARIES, Nainital, India. The ST also has a RC design with a f/13 beam at the Cassegrain focus. The AIMPOL consists of a rotatable half-wave plate (HWP), rotated from $0^{\circ}$ to $67.5^{\circ}$ in $22.5^{\circ}$ steps and a beam-splitting Wollaston prism analyser placed in the telescope beam path to produce ordinary and extraordinary images in slightly different directions separated by about 27 pixels. The imaging was done using a $1024 \times 1024$ pixel $^{2}$ CCD camera in which each pixel of

\footnotetext{
${ }^{1}$ IRAF is distributed by the national optical astronomy observatories, USA.
} 


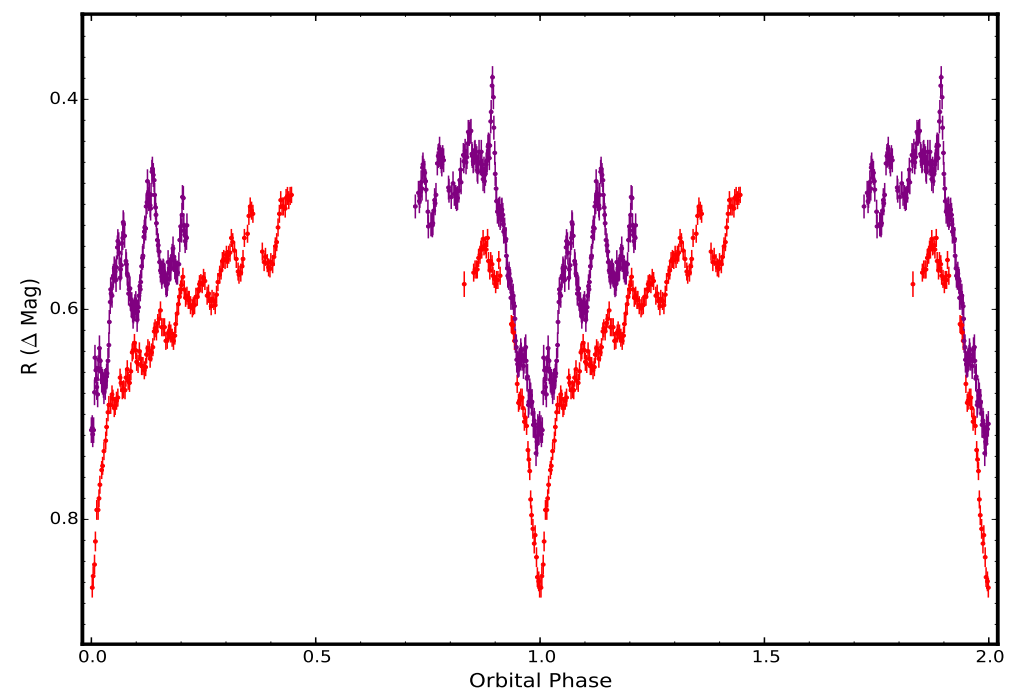

Figure 2: Orbital-phase-folded light curve of IPHAS J0258 in the R-Band on two nights of observations.

the CCD corresponds to 1.7 arcsec on the sky. The FOV is $\sim 8$ arcmin in diameter on the sky. The read-out noise and gain of the $\mathrm{CCD}$ are $7.0 e^{-}$and $11.8 e^{-}$/ADU, respectively. Data of these two sources were obtained in October, November, and December 2015 in the R-band. The observations in four positions of the HWP were used as a single data point. The full width at half-maximum (FWHM) of the stellar image varies from 2-3 pixels during the observations. The standard aperture photometry in the IRAF package was used to extract the fluxes of ordinary and extraordinary beams after bias subtraction of our program star with a good signal-to-noise ratio. By definition, the ratio $\mathrm{R}(\alpha)$ is given by

$$
R(\alpha)=\frac{I_{o}(\alpha)-I_{e}(\alpha)}{I_{o}(\alpha)+I_{e}(\alpha)}=P \cos (2 \theta-4 \alpha)
$$

where $I_{o}$ and $I_{e}$ are the intensities of the ordinary and extraordinary beams, respectively, $P$ is the fraction of total linearly polarized light, and $\theta$ is the polarization angle of the plane of polarization. Here $\alpha$ is the position of the fast axis of HWP at $0^{\circ}, 22.5^{\circ}, 45^{\circ}$, and $67.5^{\circ}$ positions with the northsouth directions.

Polarimetric standard stars HD 204827 and HD 236633 were also observed in each night to calibrate the system. In order to check the instrumental polarization, unpolarized standard stars were also observed and we obtained the value of instrumental polarization to be below $0.1 \%$ in the R-band, which is found to be constant for the past few years (e.g. Rautela et al. 2004; Pandey et al. 2009; Patel et al. 2013, 2016). The instrumental polarization was then subtracted from all the polarimetric measurements. 


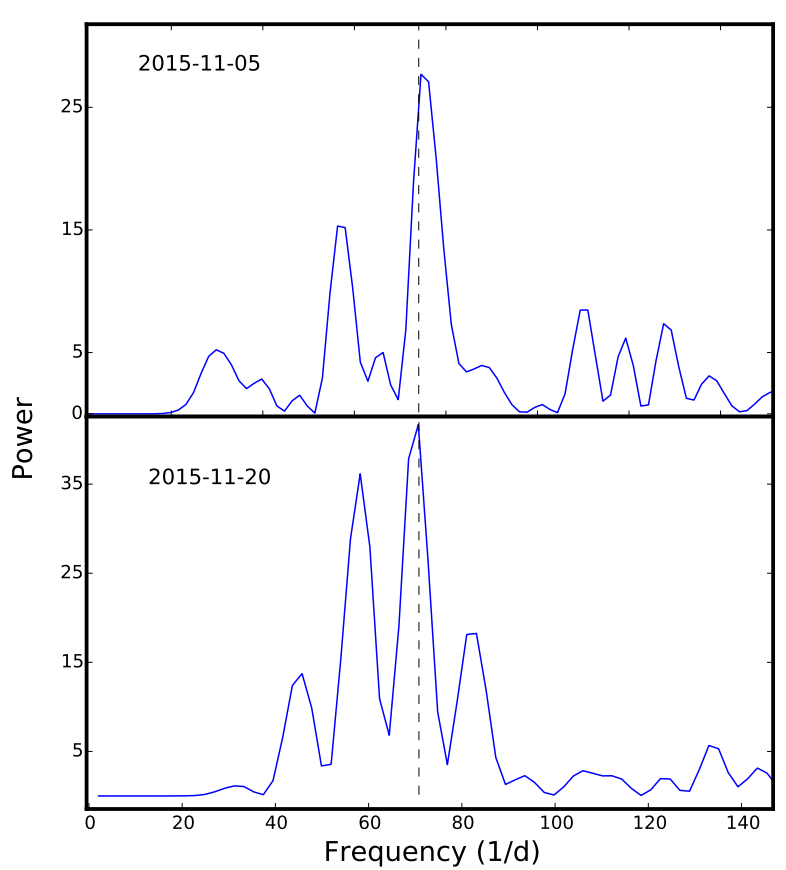

Figure 3: Power spectra of the detrended data. Detrending in the light curve was done by fitting a trigonometric polynomial of degree 2 (see text for more detail).

\section{Analysis And Results}

\subsection{IPHAS J025827.88+635234.9}

\subsubsection{Photometry}

Fig. 1 shows the R-band light curve of IPHAS J0258 as observed on two nights in November 2015. From the light curve, the eclipsing nature of this system is clear. The average eclipse depth of the two-night observations is $\sim 0.35 \mathrm{mag}$. We performed a Fourier Transform by using the Lomb-Scargle periodogram (Lomb 1976; Scargle 1982; Horne \& Baliunas 1986), but the power spectrum is noisy due to the data gap. Therefore, the CLEAN algorithm (Roberts et al. 1987) was applied to the data but due to the insufficient data length, we did not find any significant peak that corresponds to the orbital period of the system (Kozhevnikov 2014). Following Andronov \& Breus (2013), we have also fit a trigonometric polynomial of order 15 to the data with the orbital and spin frequencies. Fig. 1 shows the light curve along with this trigonometric polynomial fit. Fig. 2 shows the orbital phase folded light curves at both epochs of observations. Light curves were folded using the ephemeris $\operatorname{HJD}(\mathrm{min})$ $=2455800.42353(13)+0.245094(27) \mathrm{E}$ as given by Kozhevnikov (2014), where the numbers between brackets are the errors in units of the last digit.

In addition to the eclipses, these light curves also exhibit strong short period modulations on a time scale of $\sim 20 \mathrm{~min}$ with an average peak-to-peak amplitude of $\sim 0.06 \mathrm{mag}$ (Fig. 1 ). The detection of the He II emission line at $468.57 \mathrm{~nm}$ in the spectrum of IPHAS J0258 (Witham et al. 2007), raises the possibility that these pulsations could be due to the rotation of the WD. In order to confirm the periodicity of these pulsations, we have removed the time intervals at the minima and detrended the rest of the data using a trigonometric polynomial fit (Andronov 2012; Andronov et al. 2016). A 


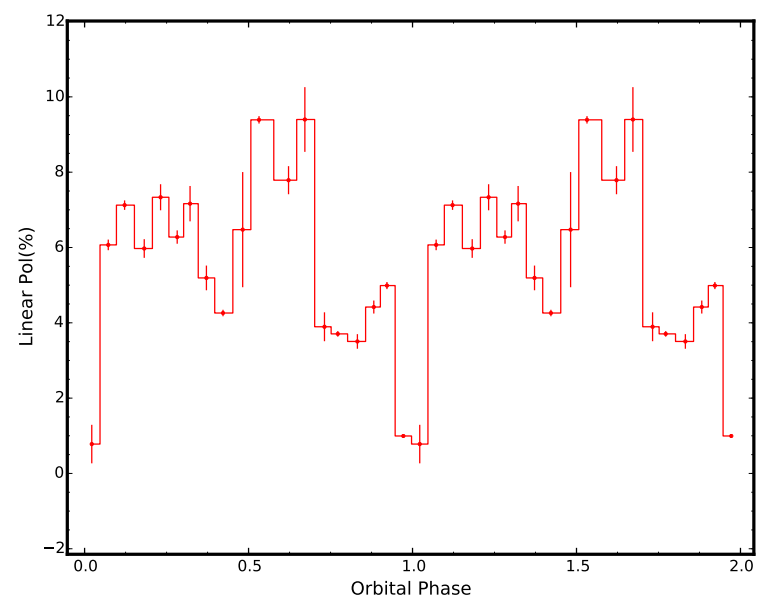

Figure 5: The degree of polarization in R-band for IPHAS J0258 shown as a function of the orbital phase in phase bins of 0.05 .

trigonometric polynomial of order two was found to be suitable to detrend the original light curve for both epochs of observations. The periodogram analysis was performed in the detrended light curves. The top and bottom panels of Fig. 3 show the Lomb-Scragle power spectra of the detrended light curves. The strongest signal was obtained at a period of 1203(25) s. The error corresponding to this period was derived by using the method given in Horne \& Baliunas (1986). The significance of this detected peak was determined by calculating the false alarm probability (Horne \& Baliunas 1986). The prominent peak was found above the 99 per cent significant level. Fig. 4 shows the spin phase folded light curves of IPHAS J0258 for both observations where the dashed line shows the best fit sine curve. The spin modulation was clearly visible in the phase folded light curves which confirms the presence of a period of 1203 s. We have also determined the mean maxima for every observing run with the sine fit which allows us to obtain one characteristic maximum for each run (Andronov et al. 1999). For epochs 5 and 20 November 2015, the resulting best-fit maxima are 2457332.32345(22) and 2457347.19032(43), respectively.

Using the eclipse width at half depth $(\Delta \phi)$, we derive the orbital inclination of the binary system using the equation (Eggleton 1983):

$$
\left(\frac{R_{2}}{a}\right)^{2}=\sin ^{2}(\pi \Delta \phi)+\cos ^{2}(\pi \Delta \phi) \cos ^{2} i
$$

where $R_{2} / a$ is the volume radius of the secondary star, which depends only on the mass ratio, $q=$ $M_{2} / M_{1}$ as

$$
\frac{R_{2}}{a}=\frac{C q^{2 / 3}}{D q^{2 / 3}+\ln \left(1+q^{1 / 3}\right)}
$$

The coefficients $C$ and $D$ are given by Eggleton (1983) as 0.49 and 0.6, respectively, for a spherical shape of Roche lobe. However, in case of CVs, the Roche lobe of secondary is definitely not spherical, having the largest size as "seen" from the white dwarf and the smallest in the polar direction. In this work, we have used the coefficients $C=0.4990$ and $D=0.5053$ (Andronov \& Andrych 2014). Using the mean empirical mass-period relation derived by Smith \& Dhillon (1998), the mass of the secondary was estimated to be $0.63 M_{\odot}$. Assuming the mean mass of the white dwarf (the primary) to be $0.85 M_{\odot}$ (Ramsay 2000), the value of $q$ was estimated as 0.74 . The ascending and descending branches of the eclipses are very different for each epoch of observations. The length of 


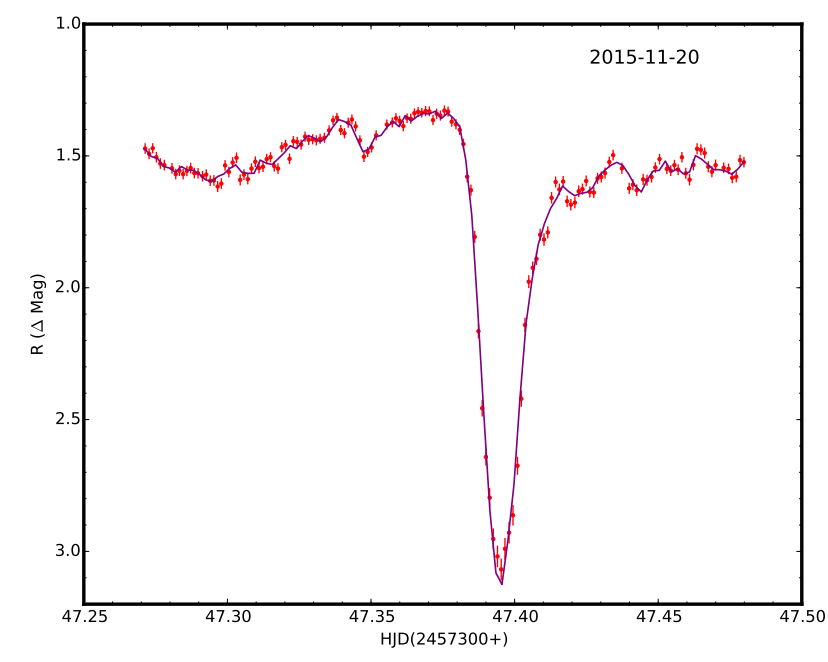

Figure 6: R-band light curve of IPHAS J0518 (red points with error bars) along with the 15 order trigonometric polynomial fit (puple line). The observed date is mentioned at the top of the panel.

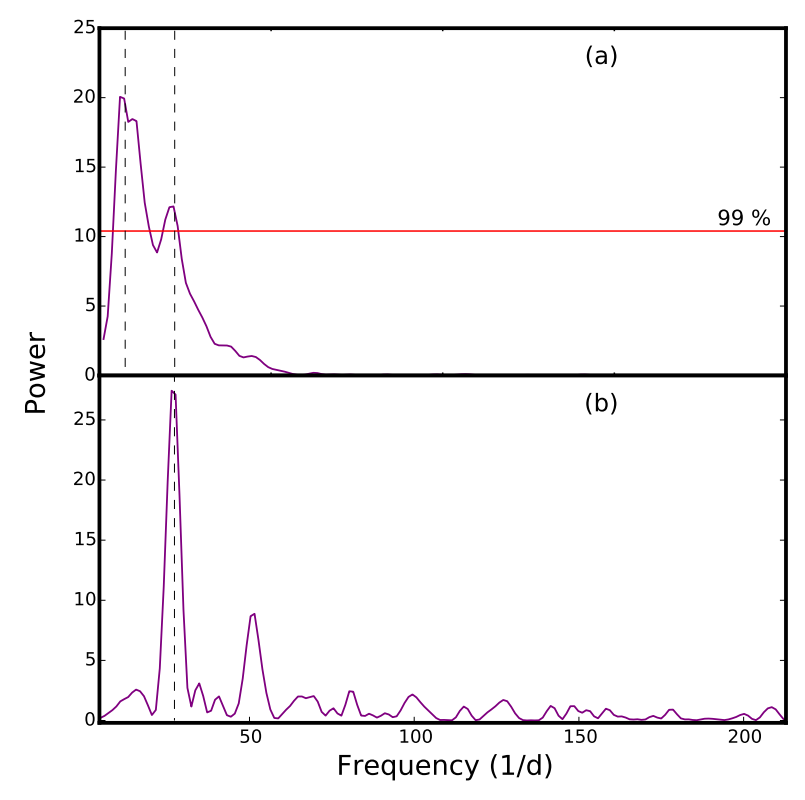

Figure 7: (a) Lomb-Scargle power spectrum of IPHAS J0518 computed from the photometric data. (b) Periodogram of the detrended light curve (see text for more detail).

the descending branches are large compared to ascending branches for every epoch of observations. Therefore, by assuming the ingress point of descending branch as a reference point, we have calculated the eclipse width at half depth. Using the observation of epoch 5 November 2015, we have estimated $\Delta \phi \sim 0.07$. In the observation of 20 November 2015, the eclipse was complex, probably due the white dwarf spin. Therefore, we have not used this for determination of $\Delta \phi$. Adopting the value of $\Delta \phi, C$, and $D$, the orbital inclination of IPHAS J0258 was estimated to be $\sim 71^{\circ}$.

\subsubsection{Linear Polarization}

The degree of polarization in the R-band was found to vary from 0.7 to $9.4 \%$ over four nights of observations. Fig. 5 shows the orbital phase-folded light curve of IPHAS J0258, which indicates that the degree of polarization was dependent on the orbital phase. The polarization value was found to be minimum near the eclipse and maximum near the phase 0.53 (i.e out of eclipse). The binsize in phase of the folded light curve was set to 0.05 .

\subsection{IPHAS J051814.33+294113.0}

\subsubsection{Photometry}

The light curve of IPHAS J0518 is shown in Fig. 6. This source also revealed a deep eclipse during which the brightness of the system dropped by $\sim 1.5$ mag. It is evident from Fig. 6 that small scale variability is present in the out of the eclipse phases. Therefore, to search for periodicities in the light curve, we performed a Fourier Transform (FT) of the photometric data using the Lomb-Scargle periodogram. The Lomb-Scargle power spectrum computed from the photometric data of IPHAS J0518 is shown in Fig. 7(a). The periods corresponding to the highest peaks in power correspond to periods 


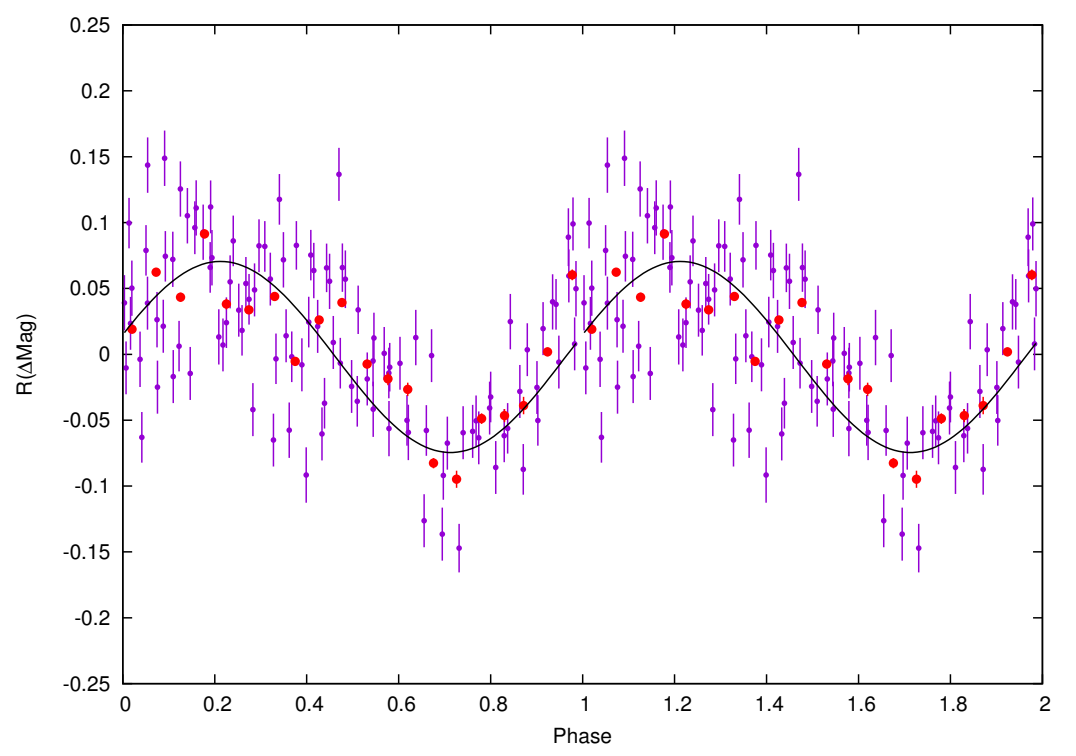

Figure 8: Folded light curve of IPHAS J0518 adopting spin period of 3277 with the best fit sine curve (full line). Solid dots shows the phase binned light curve with a binsize of 0.05 .

of 4.01(82) hrs and 3286(85) s. The longer period is similar to the timespan of the data so cannot be considered as a period of the system. To confirm the shortest period present in the power spectra, we have applied a similar approach to that applied for the period analysis of the IPHAS J0258. For detrending, we have fitted the light curve with a trigonometric polynomial of degree 2 . With this method, we have also found a prominent peak in the power spectrum that corresponds to the period 3277(81) s (Fig. 7(b)). This period could be the spin period of the source. We have also folded the detrended light curve using a spin period of $3277 \mathrm{~s}$ and an ephemeris of the minimum of the light curve (i.e first light minima) is shown in Fig. 8. The minimum was calculated to be 2457347.395(1) by fitting a parabola to the lowest part of the eclipse for this one night observing run of IPHAS J0518. For this source, we do not have a good coverage of data. Therefore, further photometric observations are required for a detailed study and to confirm its periodicity. Using a similar approach as applied to the IPHAS J0258, we calculated $\Delta \phi$ for IPHAS J0518 as $\sim 0.1$. With the orbital period as derived by Witham et al. (2007), the mass-period relationship of Smith \& Dhillon (1998) and the average mass of white dwarf of $0.85 \mathrm{M}_{\odot}$ (Ramsay et al. 2000), the value of $q$ was estimated as 0.7. Using Eqs. 2 and 3, the orbital inclination of IPHAS J0518 was estimated to be $77^{\circ}$.

\subsubsection{Linear Polarization}

The R-band polarization of IPHAS J0518 was found to be inconstant during the four nights of observations. In these observations, the R-band polarization values vary from 1 to $9 \%$.

\section{Discussion And Conclusions}

Based on short-period variations detected at periods of $1203 \mathrm{~s}$ and $3277 \mathrm{~s}$ for IPHAS J0258 and IPHAS J0518, respectively, we classify them as eclipsing IPs. In Mukai's most up-to-date distribution of IPs in the $P_{\omega}-P_{\Omega}$ plane, the majority of IPs (87\%) are found above the period gap and only a small 
fraction of IPs (13\%) lies below the period gap. The updated distribution also shows that the IPs are widely distributed over $0.25 \geq \mathrm{P}_{\omega} / \mathrm{P}_{\Omega} \geq 0.01$ (Norton et al. 2004, Gnsicke et al. 2005; and Scaringi et al. 2010). For IPHAS J0258 and IPHAS J0518, the spin-to-orbital period ratio $\left(P_{\omega} / P_{\Omega}\right)$ are derived to be $\sim 0.1$. Therefore, the derived values of orbital periods ( $\geq 3 \mathrm{hr}$ ) and $P_{\omega} / P_{\Omega}$ satisfies the condition that IPHAS J0258 and IPHAS J0518 belong to the class of IPs. The observed short period oscillations in these systems could be due to the spinning of the white dwarf. These short period oscillations are typically associated with emission either from the accretion disk or from the bright spot. The eclipse width of IPHAS J0258 was also variable in both epochs which suggest that the contribution of the accretion disk is changing in every epoch. The changes in the variations in brightness of the system, the amplitude of the spin modulation, and the morphology of the eclipse profiles can tentatively be explained by a variation in the accretion rate. A high degree of polarization is observed in both IPHAS J0258 and IPHAS J0518 systems and belongs to the class of the high magnetic field IPs.

\section{References}

Ak T., Bilir S., Ak S., Retter A. 2007, NewA, 12, 446

Allan A., Hellier C., Beardmore A. P. 1998, MNRAS, 295, 167

Andronov I. L., Arai K., Chinarova L.L. et al. 1999, AJ, 117, 574

Andronov I. L. 2012, Ap, 55, 536

Andronov I. L., Breus V. V. 2013, Ap, 56, 518

Andronov I. L., Andrych K. D. 2014, OAP, 27, 38

Andronov I. L., Tkachenko M. G., Chinarova L. L. 2016, OEJV, 176, 35

Eggleton P. P. 1983, ApJ, 268, 368

Gänsicke B. T., Marsh T. R., Edge A. et al. 2005, MNRAS, 361, 141

Hellier C., Wynn G. A., Buckley D. A. H. 2002, MNRAS, 333, 84

Horne J. H., Baliunas S. L. 1986, ApJ, 302, 757

Joshi A., Pandey J. C., Singh K. P., Agrawal P. C. 2016, ApJ, 830, 56

Kozhevnikov V. P. 2014, Ap\&SS, 349, 361

Lomb N. R. 1976, Ap\&SS, 39, 447

Norton A. J., Wynn G. A., Somerscales R. V. 2004, ApJ, 614, 349

Norton A. J., Butters O. W., Parker T. L., Wynn G. A. 2008, ApJ, 672, 524

Pandey J. C., Medhi B. J., Sagar R., Pandey A. K. 2009, MNRAS, 396, 1004

Patel M. K., Pandey J. C., Savanov I.S., Prasad V., Srivastava D. C. 2013, MNRAS, 430, 2154

Patel M. K., Pandey J. C., Karmakar S., Srivastava D. C., Savanov I. S. 2016, MNRAS, 457, 3178

Piirola V., Coyne G. V., Reiz A. 1990, A\&A, 235, 245

Ramsay G. 2000, MNRAS, 314, 403

Rautela B. S., Joshi G. C., Pandey J. C. 2004, BASI, 32, 159

Roberts D. H., Lehar J., Dreher J. W. 1987, AJ, 93, 968

Sagar R., Omar A., Kumar B. et al. 2011, CSci, 101, 1020

Scargle J. D. 1982, ApJ, 263, 835

Scaringi S., Bird A. J., Norton A. J. et al. 2010, MNRAS, 401, 2207

Schwarz R., Schwope A. D., Staude A. et al. 2007, A\&A, 473, 511

Smith D. A., Dhillon V. S. 1998, MNRAS, 301, 767

Warner B. 1995, CAS, 28, 1

Wickramasinghe D. T., Bailey J., Meggitt S. M. A. et al. 1991, MNRAS, 251, 28

Witham A. R., Knigge C., Aungwerojwit A. et al. 2007, MNRAS, 382, 1158 